\title{
Differential effects of medial septal lesions on spatial-memory tasks
}

\author{
MICHAEL W. DECKER, RICHARD J. RADEK, MARK J. MAJCHRZAK, \\ and DAVID J. ANDERSON \\ Abbott Laboratories, Abbott Park, Illinois
}

\begin{abstract}
Radiofrequency (RF) or quisqualate (QUIS) lesions of the septal area were made in rats, and acquisition of the radial-arm maze and the Morris water maze was assessed. Both RF and QUIS rats learned to find the hidden platform as quickly as sham-lesioned rats in the Morris water maze, whereas RF rats were markedly impaired and QUIS rats were mildly impaired on the radialarm maze. A spatial-discrimination version of the Morris water maze, however, revealed a deficit in RF but not QUIS rats. Interestingly, the performance of RF rats on this task was not altered by a dose of scopolamine, a muscarinic antagonist, that disrupted the performance of sham-lesioned animals. Since the relative sensitivity of these spatial tasks to disruption by septal lesions was not clearly related to spatial-mapping requirements, impaired cognitive mapping does not completely account for septal-lesion effects on memory tasks.
\end{abstract}

Much of the research on the biological basis of learning and memory in recent years has focused on the role of the hippocampal formation. The role in memory of cholinergic input to the hippocampus that arises from neurons within the medial septum and the diagonal band of Broca has been of particular interest, given the deterioration of these nuclei in Alzheimer's disease. Lesions of either the septal area or the hippocampus impair memory (for a review, see Gray \& McNaughton, 1982). Similarities in the type of impairment found in septal and hippocampal animals have led to the suggestion that damage to these two structures may be equivalent (Kesner, 1988; Olton, 1990) and that input from the septal area, much of which is cholinergic, may be essential for memory processes mediated by the hippocampus.

The involvement of the hippocampal formation in spatial learning is particularly well documented. Rats with hippocampal damage are incapable of learning spatialmemory tasks, such as the radial-arm maze and the Morris water maze. Deficits on the radial-arm maze and on the Morris water maze have also been observed in rats with septal lesions (Hagan, Salamone, Simpson, Iversen, \& Morris, 1988; Harrell, Barlow, \& Parsons, 1987; Kelsey \& Landry, 1988; Mitchell, Rawlins, Steward, \& Olton, 1982; Olton, Walker, \& Gage, 1978; Segal, Greenberger, \& Pearl, 1989). However, septal lesion effects on the water maze may be less robust. For example, Miyamoto, Kato, Narumi, and Nagaoka (1987) assessed the effects of septal lesions on water-maze and radial-maze performance in separate sets of animals, concluding that sep-

Correspondence should be addressed to $M$. W. Decker, Neuroscience Research, Pharmaceutical Products Division, Dept. 47W, AP-10, Abbott Laboratories, Abbott Park, IL 60064. tal rats were less impaired on the water maze than on the radial-arm maze. Furthermore, others have failed to observe an effect of septal lesions on the Morris water maze (Barone, Nanry, Mundy, McGinty, \& Tilson, 1991; Boitano et al., 1990; Decker, Radek, \& Pelleymounter, 1990). It is difficult to evaluate these apparent differences in the effects of septal lesions on water-maze and radialmaze performance, however, since direct, systematic comparisons of the effects of septal lesions on these two tasks are not typically attempted in the same animals. Our study, therefore, was designed to compare the effects of septal lesions on performance of these two tasks in the same animals. The effects of septal lesions were also assessed on a spatial-discrimination version of the Morris water maze.

Finally, because cholinergic dysfunction may underlie some of the behavioral deficits observed after septal lesions, we also assessed the effects of the muscarinic antagonist scopolamine and the cholinesterase inhibitor physostigmine on the spatial-discrimination version of the Morris water maze in our animals.

In this study, we compared the effects of two different lesion types-radiofrequency (RF) lesions and quisqualic acid (QUIS) lesions. Several studies have now demonstrated that QUIS lesions of the nucleus basalis magnocellularis (NBM) substantially reduces cholinergic input to cortex but does not produce the spatial-memory deficits that have been reported with NBM lesions produced by other techniques (Dunnett, Whishaw, Jones, \& Bunch, 1987; Markowska, Wenk, \& Olton, 1990; Wenk, Markowska, \& Olton, 1989). These results suggest a dissociation between the reduction of cholinergic input to cortex and spatial-memory impairments. Although this effect has been well investigated with respect to cholinergic input to cortex, similar evaluations of effects of QUIS lesions of the medial septal area have not been reported. 


\section{METHOD}

\section{Subjects}

Male Long-Evans rats (300-400 g) obtained from Charles River were used in this experiment. The rats were singly housed in a climate-controlled vivarium and allowed free access to food and water, except during radial-maze testing. The animals were maintained on a 12:12-h light:dark cycle, with testing being conducted during the light portion of the cycle.

\section{Surgical Procedures}

Medial septal lesions were produced under pentobarbital anesthesia. Ten animals received RF lesions of the medial septal area. To produce the lesions, the electrode tip was aimed at the medial septum using these coordinates: $0.5 \mathrm{~mm}$ anterior to bregma, $0.0 \mathrm{~mm}$ lateral to the midline, and $6.5 \mathrm{~mm}$ ventral to the skull surface. Radiofrequency current sufficient to maintain a temperature of $61^{\circ} \mathrm{C}$ at the electrode tip was passed for $30 \mathrm{sec}$. Quisqualic acid (Sigma) was injected into the medial septal area in 10 additional rats. One $\mu 1$ of a $0.12-\mathrm{M}$ solution of quisqualic acid in phosphate-buffered saline was injected over a 5-min period using the same coordinates as those used for RF lesions. Ten animals received sham surgery, which consisted of lowering an electrode to a point $1.0 \mathrm{~mm}$ above the target location for the lesions but passing no current.

At the end of the behavioral experiments, lesions were evaluated histologically in Nissl-stained sections and by measuring choline acetyltransferase (ChAT) activity in the dorsal hippocampus using an adaptation of the procedure of Fonnum (1975). Tissue was homogenized in $10 \mathrm{mM} \mathrm{KH}_{2} \mathrm{PO}_{4}, 10 \mathrm{mM} \mathrm{Na}{ }_{2}$ EDTA, pH 7.0, and diluted to approximately $75 \mu \mathrm{g}$ of protein per $25 \mu \mathrm{l}$ with equal volumes of $0.2 \% \mathrm{BSA}$ and $0.2 \%$ triton $\mathrm{X}-100$. Duplicate aliquots were incubated at $37^{\circ} \mathrm{C}$ for $40 \mathrm{~min}$ in a final volume of $100 \mu \mathrm{l} \mathrm{con-}$ taining $0.2 \mathrm{mM}$ [ ${ }^{3} \mathrm{H}$ ]acetyl CoA $(200,000 \mathrm{DPM}), 8 \mathrm{mM}$ choline chloride, $100 \mu \mathrm{M}$ physostigmine, $50 \mathrm{mM} \mathrm{NaH}_{2} \mathrm{PO}_{4}, 300 \mathrm{mM} \mathrm{NaCl}$, and $6.25 \mathrm{mM} \mathrm{Na}{ }_{2}$ EDTA, pH 7.4. The $\left[{ }^{3} \mathrm{H}\right]$ acetylcholine produced was isolated by two extractions with $0.5 \mathrm{ml} 2$-heptanone containing $10 \mathrm{mg} / \mathrm{ml}$ tetraphenylboron. Radioactivity was counted in $15 \mathrm{ml}$ of Insta-Gel XF (Packard). Reagent blank values were subtracted from sample DPM values. Protein values were estimated by the method of Lowry, Rosebrough, Farr, and Randall (1951) using bovine serum albumen as a standard.

\section{Behavioral Procedures}

Beginning two weeks after surgery, the rats were trained on both the Morris water maze and the radial-arm maze. Half of the rats were initially trained on the Morris water maze, and the other animals were first trained on the radial-arm maze. In addition, habituation of locomotor activity was assessed in all of the rats during the week following water-maze training. Finally, all rats were trained on the spatial-discrimination version of the Morris water maze, and the effects of scopolamine, a muscarinic antagonist, and physostigmine, an acetylcholinesterase inhibitor, were evaluated using this final task. One animal died during this final phase of behavioral testing. Data from this animal were not included in the analysis for this final phase of testing but were included in the analysis of the results of the previous phases.

Radial-arm maze. Before testing on the radial maze, the rats were gradually reduced to $85 \%$ of their free-feeding weight by restricting their food intake. The reinforcer to be used (peanut-butter chips) was introduced in their home cages during this period. Training was conducted on Monday through Friday of each week, with one training session being conducted per day. The radial maze consisted of a central octagonal platform ( $28 \mathrm{~cm}$ across) with eight identical $8.5 \times 70 \mathrm{~cm}$ arms extending from it. Training on the maze was preceded by 2 habituation days during which the rats were placed on the maze and allowed to explore freely for $10 \mathrm{~min}$. During habituation, a food reward was placed at both the midpoint and the end of each arm. After the completion of these 2 days of habituation, training sessions were conducted with food rewards available only in the food wells located at the ends of the arms. Each training session was terminated when the rat visited the ends of each of the arms or made 16 separate arm entries (entries being defined as passing the midpoint of the arm). An error was scored each time the rat entered an arm that had been previously entered during the session. Rats not completing a session within 10 min were removed from the maze and were assigned an error score by summing the number of unvisited arms and repeat entries as previously described (Decker \& Gallagher, 1987). A performance criterion of 8 different arm visits with 10 or fewer total arm entries was established a priori. The number of days required to attain this criterion on each of 3 consecutive days was determined for each animal as an index of acquisition. Animals who failed to reach criterion on 3 consecutive days during the 11 days of training were assigned the minimum score possible given their performance at the point training was concluded (i.e., the days to criterion score the animal would have received if testing had continued and no further errors were made).

Invisible-platform Morris maze. Animals were trained in a circular pool $(180 \mathrm{~cm}$ in diameter and $60 \mathrm{~cm}$ high) that had white interior walls and was filled to a depth of $37 \mathrm{~cm}$ with $26 \pm 1^{\circ} \mathrm{C}$ water. Each training trial was initiated by placing the rat in the pool facing the wall at one of four start locations equally spaced around the perimeter of the pool. A white escape platform $(13 \mathrm{~cm}$ in diameter) was placed in the center of one of the quadrants defined by the start locations. The platform was submerged $1.0 \mathrm{~cm}$ below the water's surface and rendered invisible by the addition of powdered milk to the water. Each trial ended when the rat found and climbed onto the escape platform, and the latency to escape was recorded by the experimenter. If the rat did not find the platform within $90 \mathrm{sec}$, it was guided to the platform by the experimenter and $90 \mathrm{sec}$ was recorded as the escape latency. In either case, the rat remained on the platform for $20 \mathrm{sec}$. Four such place-training trials were conducted each day, divided into two blocks of two trials each. Trials within a block were separated by a 5- to 10 -sec intertrial interval, and trial blocks were separated by 3 to $4 \mathrm{~min}$. The start locations were varied so that one trial in each block was started from one of the two start locations nearer the platform and one was started from one of the locations farther from the platform. No start location was used more than once on any training day. Training was conducted for 4 days. On the 5th day, a 60 -sec free-swim probe trial was conducted with the escape platform removed from the maze. This trial was recorded on videotape and later analyzed by measuring the amount of time each rat spent in the training quadrant (the quadrant of the pool in which the platform had been located during training).

Spatial-discrimination Morris water maze. In this version of the water maze, two platforms were used. Each platform was covered with aluminum foil and protruded $1.5 \mathrm{~cm}$ above the water surface, but one of the platforms was made of expanded polystyrene and floated on the surface of the water. Thus, only one of the platforms provided a means of escape from the water. The locations of the platforms remained fixed during a training session. An error was recorded each time the rat contacted the floating platform with its head or forepaws. If the rat swam away from the floating platform and subsequently made contact with it again during the trial, an additional error was recorded (i.e., more than one error was possible during a trial). Each trial ended when the rat found the escape platform. Occasionally, a rat would not find the correct platform within $90 \mathrm{sec}$ and had to be guided to it. In these cases, the total errors committed in the 90 -sec period were recorded, or in the very rare event that neither platform was contacted, an error score of 1 was arbitrarily assigned. During initial training, six trials per day were conducted, with the starting location being varied across trials. 
Following the initial training period, the rats received a series of trials during which the effects of scopolamine $\mathrm{HBr}(0.3 \mathrm{mg} / \mathrm{kg})$ and physostigmine $(0.06 \mathrm{mg} / \mathrm{kg})$ were assessed. Drug effects were assessed during 20-trial sessions conducted on Mondays and Thursdays. Ten minutes prior to each of these test sessions, drug or saline was injected i.p. On the day after each test session, a "recovery" session was conducted during which no drugs were injected and the rats were given 10 additional trials using the same platform locations used during the previous test session. One drug and one saline session was conducted for each animal during the week in a counterbalanced order. Half of the animals received scopolamine during the first week of testing followed by a week of no training before being tested under physostigmine. The other animals were first tested under physostigmine. For each drug or saline session, a new set of platform locations was used. Thus, a total of five pairs of platform locations were used during these experiments: one for initial training, one each for the physostigmine session and its control session, and one each for the scopolamine session and its control session.

Habituation of locomotor activity. Locomotor activity was measured in a $41 \times 41 \mathrm{~cm}$ open field using Digiscan activity monitors (Omnitech Electronics, Columbus, $\mathrm{OH}$ ). On the 1st day, the rats were placed in this novel environment and horizontal activity was recorded in 1-min time bins for a total of $5 \mathrm{~min}$. Twenty-four hours later, the rats were placed in the open field for an additional 5-min session.

\section{Drugs}

(-)Scopolamine $\mathrm{HBr}$ and physostigmine (eserine) hemisulfate (Sigma) were dissolved in sterile $0.9 \%$ saline. The dose of physostigmine $(0.06 \mathrm{mg} / \mathrm{kg})$ was based on the weight of the free base, whereas the dose of scopolamine $(0.3 \mathrm{mg} / \mathrm{kg})$ was based on the weight of the salt. Sterile $0.9 \%$ saline $(1.0 \mathrm{ml} / \mathrm{kg})$ was used in control injections.

\section{Statisties}

The data were evaluated using analyses of variance (ANOVAs). Post-hoc pairwise comparisons were evaluated using Fisher's protected least significant difference test.

\section{RESULTS}

\section{Lesion Verification}

As can be seen in Figure 1, both lesion techniques reduced ChAT activity in the dorsal hippocampus. ChAT activity was reduced by $34.4 \%$ in rats receiving intraseptal QUIS $(p<.0001)$ and by $74.4 \%$ in rats receiving RF lesions of the septal area $(p<.0001)$. Although both lesions produced substantial reductions in ChAT activity, the RF lesions were significantly more effective than the QUIS lesions $(p<.0001)$.

Histological examination revealed extensive damage to the medial septum in rats receiving RF lesions. Damage typically extended into a substantial portion of the lateral septum and into the vertical limb of the diagonal band of Broca below the level of the anterior commissure. The lesion, however, did not extend ventrally into the decussation of the anterior commissure at more posterior levels, and in most animals no damage to the corpus callosum was observed. Damage was typically bilateral, but some animals had a lesion that was more complete on one side. RF lesions produce rather clearly defined tissue destruction, but QUIS-induced damage is restricted to neurons.
ChAT ACTIVITY IN DORSAL HIPPOCAMPUS

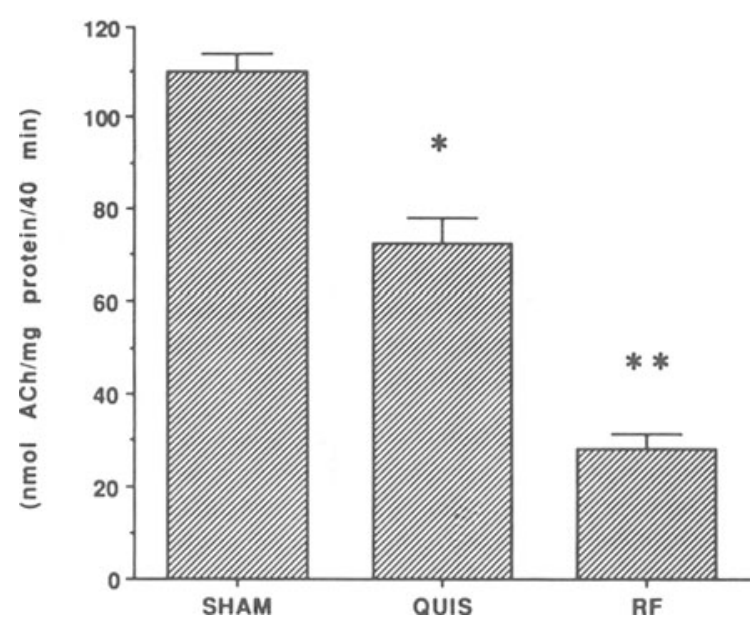

Figure 1. Choline acetyltransferase (ChAT) activity in the dorsal hippocampus of rats with sham, quisqualic acid (QUIS), or radiofrequency (RF) lesions of the medial septal area. DDiferent from sham, $p<.0001$. *Different from sham and QUIS, $p<.0001$.

Thus, it is somewhat difficult to determine the relative extent of these two lesions histologically. Cell loss in the medial septal area was apparent in QUIS-lesioned rats. This damage appeared to be restricted to the more medial aspects of the septum and did not seem to extend ventrally into the vertical limb of the diagonal band of Broca as much as it did with RF lesions.

\section{Behavior}

Radial-arm maze. Analysis of the errors during acquisition of the radial maze revealed a significant effect of lesion $[F(2,27)=22.56, p<.0001$; Figure 2]. Both groups of lesioned animals made more errors during training on the radial-arm maze than did controls $(p<.0001$ for RF-lesioned rats and $p<.05$ for QUIS-lesioned rats), although the rats with RF lesions of the septal area made substantially more errors than did the rats with QUIS lesions. All control rats had at least three consecutive days of criterion performance and took fewer days on average to attain this level of performance (6.8 \pm 0.8 days) than did either rats with QUIS lesions $(9.6 \pm 1.3$ days, $p<$ $.05)$ or rats with $R F$ lesions $(13.6 \pm 0.2$ days, $p<.0001)$.

Invisible-platform Morris maze. Evaluation of mean escape latencies during training on the Morris water maze (Figure 3) failed to reveal an overall lesion effect $[F(2,27)$ $=0.75, p>.40$ ], although the lesion $\times$ training block interaction was statistically significant $[F(7,189)=1.82$, $p<.04]$. This interaction appears to be related to significantly higher escape latencies exhibited by animals in both lesion groups compared with control animals during the first training block ( $p s<.05)$ contrasted with the lack of group differences found during subsequent training blocks. The results of the free-swim probe trial (Figure 4) reveal no effect of RF lesions compared with control per- 
RADIAL MAZE ACQUISITION

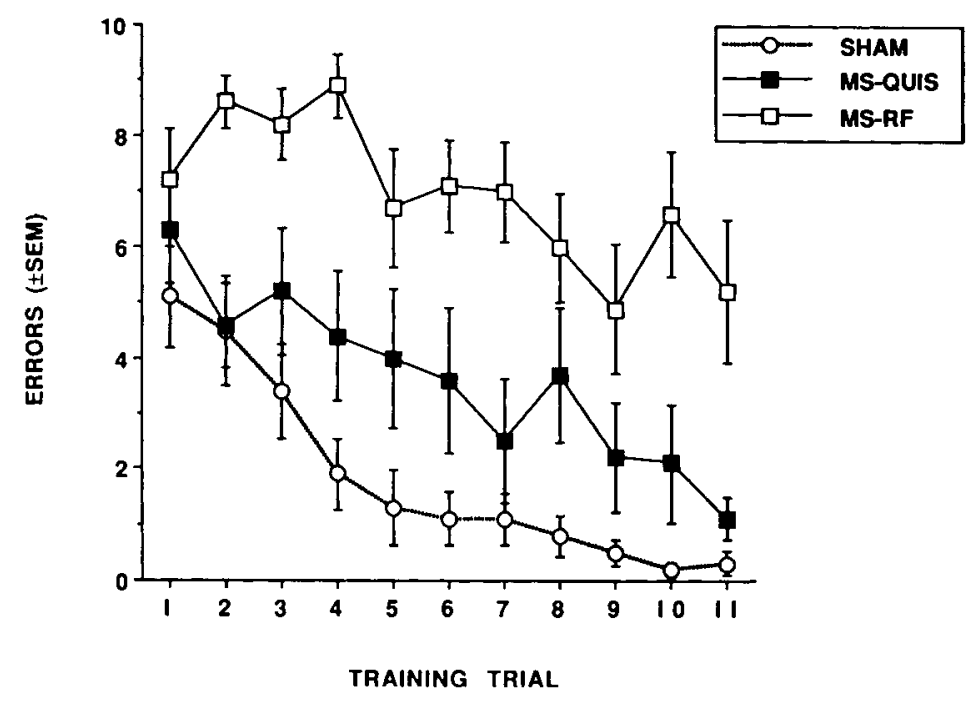

Figure 2. Errors during radial-arm-maze training for rats with sham, quisqualic acid (QUIS), or radiofrequency (RF) lesions of the medial septal area. One training trial was conducted per day. QUIS lesions slightly impaired and RF lesions markedly impaired performance. See text for statistical analysis.

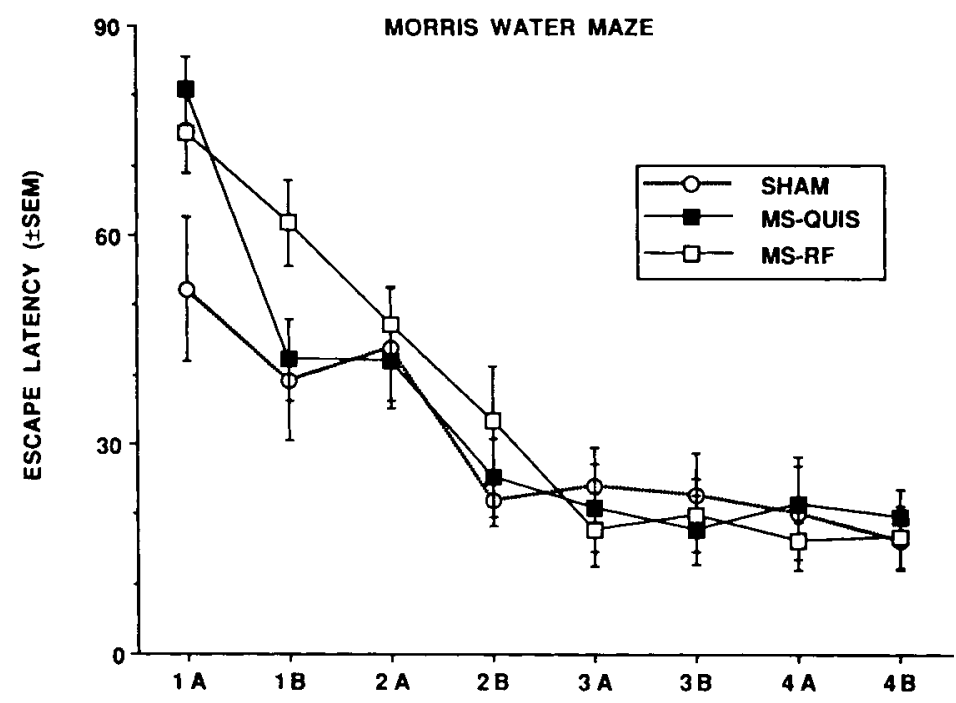

TRAINING BLOCK

Figure 3. Mean escape latencies during training on the invisible-platform Morris water maze for rats with sham, quisqualic acid (QUIS), or radiofrequency (RF) lesions of the medial septal area. Two training blocks of two trials each were conducted per day. Septal lesions did not significantly impair performance. See text for statistical analysis. 


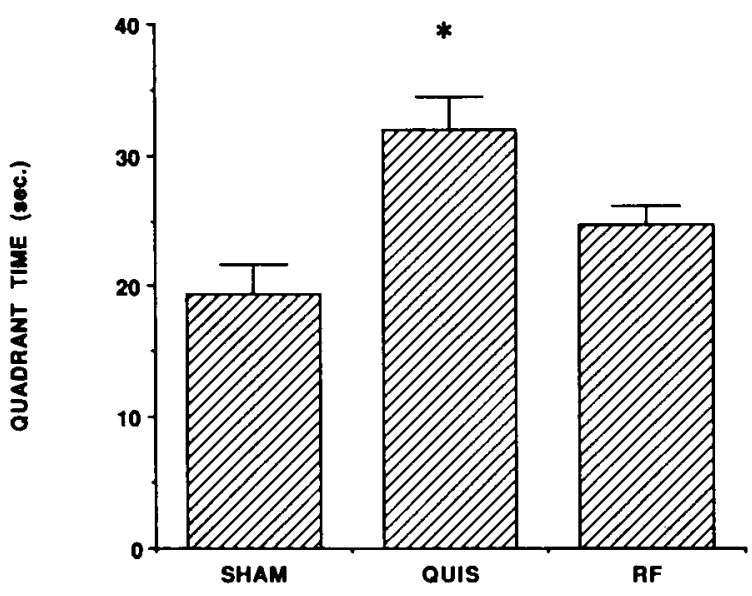

Figure 4. Time spent in the training quadrant during a 60-sec free swim conducted $24 \mathrm{~h}$ after the last training trial depicted in Figure 3. The escape platform had been located in the middle of this quadrant during training but was not present in the pool during the free swim. *Different from sham, $p<.05$.

formance and show that QUIS-treated animals actually spent somewhat more time in the training quadrant than did control rats $(p<.05)$.

Spatial-discrimination Morris water maze. In contrast to the results obtained with the invisible-platform Morris water maze, a significant lesion effect was obtained with the spatial-discrimination version of the Morris water maze $[F(2,27)=13.46, p<.0001]$. As can be seen in Figure 5, animals who had received RF lesions of the septal area made significantly more errors than did either control animals or animals that received QUIS injections into the septal area. The latter two groups did not differ significantly on this measure.

Cholinergic drug effects on spatial discrimination. The effects of scopolamine on spatial discrimination were evaluated using a three-way repeated measures ANOVA, with lesion as the between-group measure and scopolamine as a repeated measure. In addition, the 20-trial training session was divided into two 10-trial blocks and these two blocks were treated as a second repeated measure. As would be expected, the rats made fewer errors during the second of the two training blocks conducted on the test day, resulting in a significant training-block effect $[F(1,26)=115.67, p<.0001]$. In addition, our analysis revealed a significant disruptive effect of scopolamine $[F(1,26)=4.50, p<.05]$. Interestingly, as can be seen in Figure 6, scopolamine increased errors among sham-treated and QUIS-injected animals but did not appear to alter the performance of rats with RF lesions of the septal area. In contrast to the results obtained with scopolamine, physostigmine did not significantly alter performance $[F(1,26)=1.56, p>.2]$ in any of the groups (data not shown).

Habituation of locomotor activity. Analysis of horizontal activity counts (Figure 7) during the two 5-min sessions in the open field was conducted using a repeated measures ANOVA, with minutes within each session and session day as the repeated measures and lesion as a between-group factor. The effect of lesion was statistically significant $[F(2,27)=4.78, p<.02]$, primarily because animals with RF lesions did not display the within-session habituation of locomotor activity exhibited by control animals [minute $\times$ lesion interaction, $F(8,108)$ $=3.89, p<.001]$. All groups tended to decrease activity across days $[F(1,27)=53.46, p<.0001]$, but this effect was less pronounced in the lesion groups (particularly the QUIS lesion group), resulting in a significant lesion $\times$ day interaction $[F(2,27)=3.74, p<.04]$.

\section{DISCUSSION}

In the current study, RF septal lesions profoundly impaired and QUIS lesions mildly impaired performance on the radial maze, whereas neither lesion significantly impaired performance on the standard version of the Morris water maze. The spatial-discrimination version of the water maze was of intermediate sensitivity, revealing a deficit only in rats with RF lesions.

Our finding that RF lesions of the septal area impaired performance on the radial maze is consistent with literature reports that septal lesions produce pronounced, longlasting deficits on this task (Harrell et al., 1987; Mitchell et al., 1982; Olton et al., 1978). The impaired radialmaze performance we found with QUIS lesions of the septal area contrasts with the normal radial-maze performance we have observed in rats with QUIS lesions of cholinergic neurons in the nucleus basalis (unpublished data). Several other recent reports have failed to detect deficits in

SPATIAL DISCRIMINATION MORRIS WATER MAZE

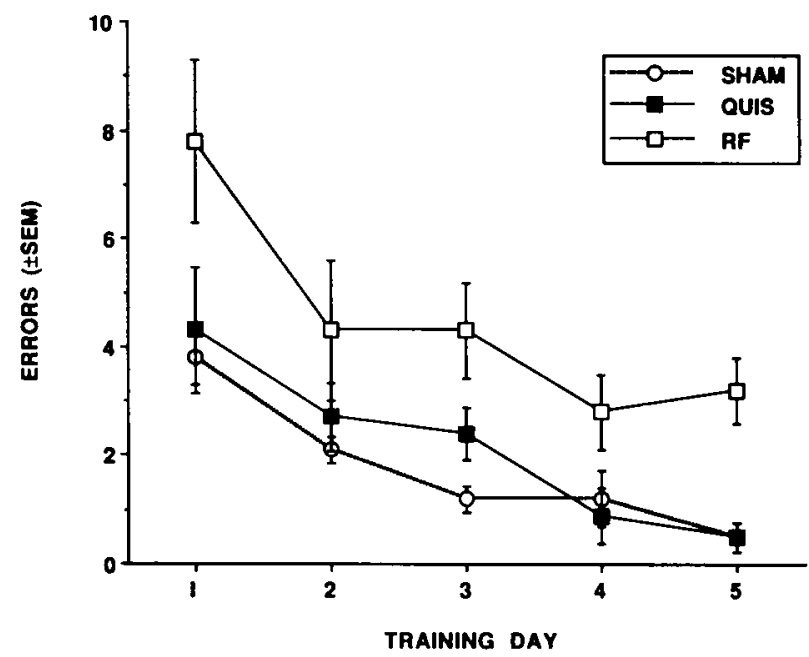

Figure 5. Errors during spatial-discrimination Morrts-water-maze training for rats with sham, quisqualic acid (QUIS), or radiofrequency (RF) lesions of the medivl septal area. Six training trials were conducted per day. RF lesions morked impoired performance, but QUIS lesions did not. See text for statistical analysis. 


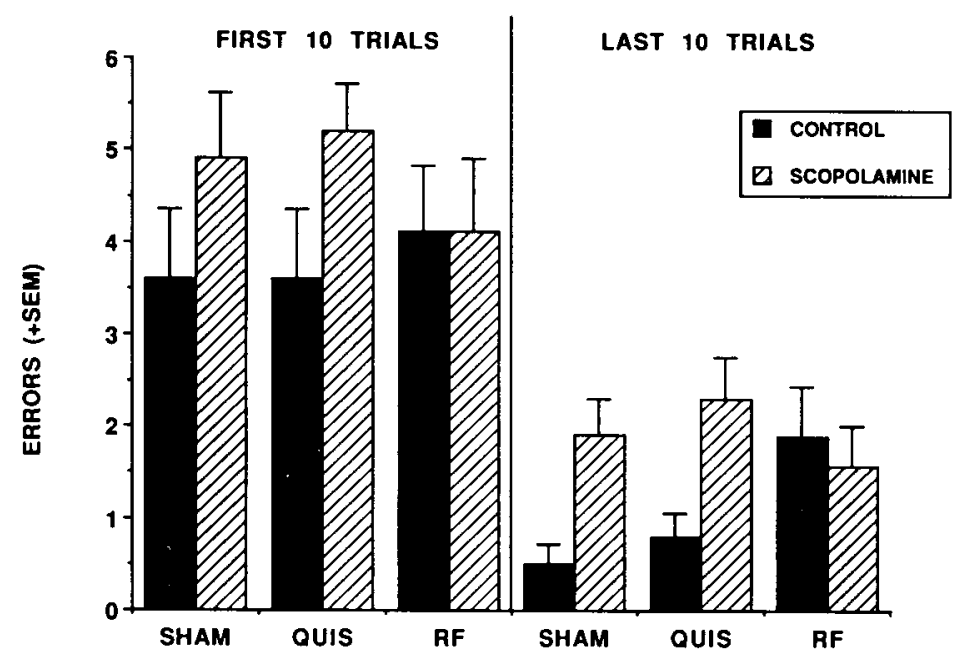

Figure 6. The effects of scopolamine $(0.3 \mathrm{mg} / \mathrm{kg}$, i.p.) on spatial-discrimination performance in rats with sham, quisqualic acid (QUIS), or radiofrequency (RF) lesions of the medial septal area. Each rat received one session (20 trials) under saline and one under scopolamine. Scopolamine impaired the performance of rats with sham or QUIS lesions but did not affect the performance of rats with RF lesions. See text for statistical analysis.

other spatial tasks with QUIS injections into the NBM, despite the profound reduction of cholinergic markers in the cortex that these injections produce (Dunnett et al., 1987; Markowska et al., 1990; Wenk et al., 1989). Thus, the behavioral effects we observed with QUIS lesions of the septal area may be suggestive of important differences between cholinergic neurons in the nucleus basalis and the septal area.

Our failure to observe an effect of septal lesions on standard-water-maze performance contrasts with other reports of deficits in Morris-water-maze performance in rats with septal lesions (Hagan et al., 1988; Kelsey \& Landry, 1988; Riekkinen, Sirviö, \& Riekkinen, 1990; Segal et al., 1989). However, these reported effects are relatively mild compared with the impairments noted with direct damage to the hippocampus (Morris, 1983), and it has also been noted that the deficits on the water maze

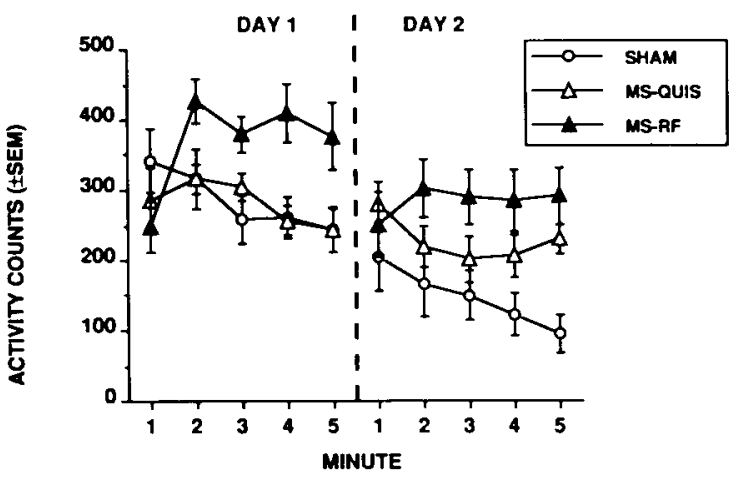

Figure 7. Locomotor activity of rats with sham, quisqualic acid (QUIS), or radiofrequency (RF) lesions of the medial septal area. Rats were exposed to the open field for $5 \mathrm{~min}$ on each of 2 consecutive days. See text for statistical analysis. associated with septal damage are less severe than those associated with damage to the fimbria-fornix (Segal et al., 1989; Sutherland \& Rodriguez, 1989) or to the NBM (Miyamoto et al., 1987; Riekkinen et al., 1990; but see Hagan et al., 1988). Furthermore, as noted in the introduction, other studies have also failed to observe acquisition deficits on the standard version of the Morris water maze in rats with septal lesions (Barone et al., 1991; Boitano et al., 1990; Decker et al., 1990). Although we do not dispute the results of studies demonstrating septallesion-induced impairment of Morris-water-maze performance, the results of the current study do suggest that the radial-arm maze may be more sensitive to septohippocampal dysfunction than is the Morris water maze.

It is unlikely that the differences in the sensitivity of the radial maze and the water maze to septal damage reflect a greater sensitivity of the radial maze to brain damage in general since medial frontal cortex lesions have been reported to produce the opposite pattern of results-profound impairments on the Morris water maze and relatively mild, transient deficits on the radial-arm maze (Kolb, Sutherland, \& Whishaw, 1983).

Differences we observed in task sensitivity to septallesion effects are most likely related to the informationprocessing requirements of the tasks. The radial maze is typically regarded as a working spatial-memory task in that the information to be remembered is trial dependent since a different set of arms is visited on each trial. In contrast, the location of the escape platform in the Morris water maze remains constant across trials, and this task is typically regarded as a reference spatial-memory task. The differences we found in the effects of septal lesions on the performance of these two tasks, then, could result from greater effects of septal lesions on working memory, an interpretation consistent with the conclusions 
reached by Miyamoto et al. (1987). Olton (1983) has argued that the hippocampus is critical for working memory, although others have argued that the hippocampus is important for all forms of spatial memory (Morris, 1983; Nadel \& MacDonald, 1980). However, it is still possible that the septal input to the hippocampus is more important in working-memory spatial tasks than it is in reference-memory spatial tasks. Perhaps consistent with this suggestion is the recent demonstration that reversible inactivation of the septal area profoundly impairs radial-arm-maze performance in rats (Mizumori, Perez, Alvarado, Barnes, \& McNaughton, 1990) but does not alter the place-field selectivity of neurons in CAl of the hippocampus (Mizumori, McNaughton, Barnes, \& Fox, 1989). Furthermore, training on reference- and working-memory radial-maze tasks produces different patterns of activation of the septohippocampal cholinergic system (Durkin, Toumane, \& Jaffard, 1988), which suggests that the septohippocampal cholinergic system may be differentially involved in these two forms of memory.

Although differential involvement of the septal area in working and reference memory might address differences in the effects of septal lesions on radial-maze and hiddenplatform water-maze performance, it does not account for differences in the effects of our septal lesions on performance of the two versions of the Morris water maze. Rats with RF lesions of the septal area were profoundly impaired on the spatial-discrimination water maze but unimpaired on the hidden-platform task, even though both of these tasks are regarded as reference-memory tasks. The animals in this study were all trained on the hiddenplatform version of the water maze before they were trained on the spatial-discrimination task. In pilot studies, however, we observed similar lesion-induced spatialdiscrimination deficits in rats with no previous experience in the water maze. Thus, it is unlikely that interference resulting from previous experience in the hidden-platform task can account for the deficits observed on the spatialdiscrimination task in the current study.

Although the spatial-processing requirements of these two water-maze tasks are also quite similar, the spatialdiscrimination version of the water maze that revealed a lesion effect in the current study would seem to be somewhat less demanding. Rats are required to learn to find the specific location of a hidden platform in the standard water maze, whereas they are required only to learn which of two platform locations is the correct spatial location in the spatial-discrimination task. However, in the spatialdiscrimination task, the availability of two identical, salient local cues-one designating the correct location and one designating an incorrect location-may actually have made the task more difficult for septal animals. These salient cues might be regarded as distractors, since to perform this task correctly, the animal must be able to shift its attention away from these local cues to extramaze cues. Since septal rats have difficulty in learning complex tasks in the presence of distracting stimuli (Gray, 1982; Isaac- son, 1982), the presence of two platforms in the spatialdiscrimination water maze might have contributed to the greater impairment we observed on this task. Attentional deficits might also partly account for the very poor radialmaze performance we observed in these animals.

In the current study, the open-field locomotor activity of rats with RF lesions, unlike that of sham-lesioned rats, did not decrease over time. Habituation deficits have often been reported with septal damage (Gray \& McNaughton, 1982), and the within-session deficits in habituation of open-field locomotor activity we observed with RF lesions are similar to those that have also been reported for maze and object exploration (Herrmann, Poucet, \& Ellen, 1985; Poucet, 1989). These habituation effects have been attributed to faulty processing of spatial information (Herrmann et al., 1985; Poucet, 1989) or reduced emotionality (Gray \& McNaughton, 1982). It would seem that attentional deficits could also contribute to the effects of RF septal lesions on habituation.

Comparison of RF- and QUIS-lesion effects suggests that RF lesions were more disruptive in the tasks assessed in the current study. Rats with RF lesions were profoundly impaired on the radial-arm maze and significantly impaired in the spatial-discrimination water maze, whereas rats with QUIS lesions were more mildly impaired on the radial maze and were not significantly impaired in the spatial-discrimination water maze. Furthermore, RF lesions disrupted short-term, within-session habituation of activity in the open field, whereas QUIS lesions only affected the long-term habituation of activity observed when animals were returned to the same open field $24 \mathrm{~h}$ later. Thus, the significantly greater depletion of ChAT activity found with RF lesions was associated with more pronounced behavioral impairments in the spatial tasks. It must be acknowledged, however, that RF lesions also produce greater tissue damage, particularly with respect to fibers of passage.

This relationship between decreases in ChAT activity produced by the two types of lesions and behavioral effects is consistent with the hypothesis that disruption of cholinergic input to the hippocampus is of central importance in the memory effects associated with septal damage. Given the rather extensive destruction of the diagonal band of Broca observed with RF lesions, it is likely that cholinergic input to those cortical regions (particularly cingulate and entorhinal cortex) that receive input from the diagonal band (Jacobowitz \& Creed, 1983; Kristt, McGowan, Martin-MacKinnon, \& Solomon, 1985; Saper, 1984) may also have been affected more by RF lesions than by QUIS lesions. In addition, damage to other neurons within the septal area, particularly GABAergic neurons that project to the hippocampus (Kohler, Chan-Palay, \& Wu, 1984), may have played an important role in the effects we observed.

Our finding that scopolamine disrupted the spatialdiscrimination performance of sham-lesioned rats but did not further impair the spatial-discrimination performance of rats with RF lesions of the septal area is, perhaps, some- 
what surprising. It might have been predicted that an animal with a compromised septohippocampal cholinergic system would have been more rather than less sensitive to cholinergic blockade. Our RF lesions were rather complete, so perhaps the animals were unable to make use of this system in learning the task. If the animals learned the task (albeit less well) using an alternative, noncholinergic system, it would not be unreasonable to find cholinergic blockade to be less effective in disrupting performance. An alternative interpretation that scopolamine failed to affect RF-lesioned animals because they were already maximally impaired is not supported by the evidence. In this experiment, even rats with RF lesions showed significant improvement across training blocks and were performing at better-than-chance levels by the second training block. Yet even during this latter stage of training, their performance was unaffected by scopolamine. Similarly, scopolamine effects on radial-maze performance are attenuated in rats with lesions of the dentate gyrus (Tilson et al., 1988). One interpretation of these two sets of findings is that the septohippocampal system is the principal target for the disruption of spatial memory produced by muscarinic blockade, a view that is consistent with previous reports of spatial-memory deficits resulting from intraseptal manipulation of septohippocampal cholinergic activity (Bostock, Gallagher, \& King, 1988; Brioni, Decker, Gamboa, Izquierdo, \& McGaugh, 1990; Chrobak, Stackman, \& Walsh, 1989; Givens \& Olton, 1990; Mizumori et al., 1990).

In general, our results are consistent with the participation of septal afferents in some aspects of hippocampal memory processing. However, septal input to the hippocampus may not be absolutely necessary for spatial navigation, since septal rats were not significantly impaired in the hidden-platform Morris water maze in our study. Thus, it would appear that septal input may not be equally important in all hippocampal memory functions. Further research will be necessary to distinguish the relative importance of the septohippocampal pathway in different forms of memory and to elucidate the memory-modulatory role of the septal area.

\section{REFERENCES}

Barone, S., Jr., Nanry, K. P., Mundy, W. R., McGinty, J. F., \& TILSON, H. A. (1991). Spatial learning deficits are not solely due to cholinergic deficits following medial septal lesions with colchicine. Psychobiology, 19, 41-50.

Boitano, J. J., Dokla, C. P. J., Parker, S., Stalzer, K., NoRELLI, N., \& FIORINI, M. (1990). Effects of medial septal lesions on activity and water maze performance. Society for Neuroscience $A b$ stracts, 16, 1248.

Bostock, E., Gallagher, M., KING, R. A. (1988). Effects of opioid microinjections into the medial septal area on spatial memory in rats. Behavioral Neuroscience, 102, 643-652.

Brioni, J. D., Decker, M. W., Gamboa, L. P., Izquierdo, I., * MCGAUGH, J. L. (1990). Muscimol injections in the medial septum impair spatial learning. Brain Research, 522, 227-234.

Chrobak, J. J., Stackman, R. W., \& Walsh, T. J. (1989). Intraseptal administration of muscimol produces dose-dependent memory impairments in the rat. Behavioral \& Neural Biology, 52, 357-369.
Decker, M. W., \& Gallagher, M. (1987). Scopolamine-disruption of radial arm maze performance: Modification by noradrenergic depletion. Brain Research, 417, 59-69.

Decker, M. W., Radek, R. J., \& Pelleymounter, M. A. (1990). Differential effects of medial septal lesions on acquisition in two spatial memory tasks. Society for Neuroscience Abstracts, 16, 1246.

DunnetT, S. B., Whishaw, I. Q., Jones, G. H., \& Bunch, S. T. (1987). Behavioural, biochemical and histochemical effects of different neurotoxic amino acids injected into nucleus basalis magnocellularis of rats. Neuroscience, 20, 653-669.

Durkin, T., Toumane, A., \& JAFFARD, R. (1988). Differential hippocampal and cortical cholinergic activation as a function of both type and duration of memory testing of mice in a radial maze. Society for Neuroscience Abstracts, 14, 396.

FonNum, F. (1975). A rapid radiochemical method for the determination of choline acetyltransferase. Journal of Neurochemistry, 24, 407-409.

Grvens, B. S., \& Olton, D. S. (1990). Cholinergic and GABAergic modulation of medial septal area: Effect on working memory. Behavioral Neuroscience, 104, 849-855.

GraY, J. A. (1982). The neuropsychology of anxiety. New York: Oxford University Press.

Gray, J. A., McNaughton, N. (1982). Comparison between the behavioural effects of septal and hippocampal lesions: A review. Neuroscience \& Biobehavioral Reviews, 7, 119-188.

hagan, J. L., Salamone, J. D., Simpson, J., Iversen, S. D., \& Morris, R. G. M. (1988). Place navigation in rats is impaired by lesions of medial septum and diagonal band but not nucleus basalis magnocellularis. Behavioural Brain Research, 27, 9-20.

Harrel., L. E., Barlow, T. S., \& Parsons, D. (1987). Cholinergic neurons, learning and recovery of function. Behavioral Neuroscience, 101, 644-652.

Herrmann, T., Poucet, B., Ellen, P. (1985). Spatial problem solving in a dual runway task by normal and septal rats. Behavioral Neuroscience, 99, 631-637.

IsAACSON, R. L. (1982). The limbic system (2nd ed.). New York: Plenum.

JACOBowitz, D.M., \& Creed, G. J. (1983). Cholinergic projection sites of the nucleus of tractus diagonalis. Brain Research Bulletin, 10, 365-371.

KELSEY, J. E., \& LANDRY, B. A. (1988). Medial septal lesions distupt spatial mapping ability in rats. Behavioral Neuroscience, 102, 289-293.

KESNER, R. P. (1988). Reevaluation of the contribution of the basal forebrain cholinergic system to memory. Neurobiology of Aging, 9, 609-616.

Kohler, C., Chan-Palay, V., Wu, J. (1984). Septal neurons containing glutamic acid decarboxylase immunoreactivity project to the hippocampal region in the rat brain. Anatomy \& Embryology, 169, $41-44$.

Kolb, B., Sutherland, R. J., \& Whishaw, I. Q. (1983). A comparison of the contributions of the frontal and parietal association cortex to spatial localization in rats. Behavioral Neuroscience, 97, 13-27.

KristT, D. A., McGowan, R. A., JR., Martin-MacKinnon, N., * SoLomon, J. (1985). Basal forebrain innervation of rodent neocortex: Studies using acetylcholinesterase histochemistry, Golgi, and lesion strategies. Brain Research, 337, 19-39.

Lowry, O. H., Rosebrough, N. J., Fark, A. L., \&andall, R. J. (1951). Protein measurement with the Folin phenol reagent. Journal of Biological Chemistry, 193, 265-275.

Markowska, A. L., Wenk, G. L., Olton, D. S. (1990). Nucleus basalis magnocellularis and memory: Differential effects of two neurotoxins. Behavioral \& Neural Biology, 54, 13-26.

Mitchell, S., Rawlins, J., Steward, O., \& Olton, D. S. (1982). Medial septal area lesions disrupt rhythm and cholinergic staining in medial entorhinal cortex and produce impaired radial arm maze behavior in rats. Journal of Neuroscience, 2, 292-302.

Miyamoto, M., Kato, J., Narumi, S., \& Nagaoka, A. (1987). Characteristics of memory impairment following lesioning of the basal forebrain and medial septal nucleus in rats. Brain Research, 419, 19-31.

Mizumori, S. J. Y., McNaughton, B. L., BARnes, C. A., \& Fox, K. B. (1989). Preserved spatial coding in hippocampal CA1 pyrami- 
dal cells during reversible suppression of CA3c output: Evidence for pattern completion in hippocampus. Journal of Neuroscience, 9 , 3915-3928

Mizumori, S. J. Y., Perez, G. M., Alvarado, M. C., Barnes, C. A., \& McNaughton, B. L. (1990). Reversible inactivation of the medial septum differentially affects two forms of learning. Brain Research, $528,12-20$

Morris, R. G. M. (1983). An attempt to dissociate "spatial-mapping' and "working-memory" theories of hippocampal function. In W. Seifert (Ed.), Neurobiology of the hippocampus (pp. 405-432). London: Academic Press.

Nadel, L., MacDonald, L. (1980). Hippocampus: Cognitive map or working memory? Behavioral \& Neural Biology, 29, 405-409.

Olton, D. S. (1983). Memory functions and the hippocampus. In W. Seifert (Ed.), Neurobiology of the hippocampus (pp. 335-373). London: Academic Press.

OLtoN, D. S. (1990). Dementia: Animal models of the cognitive impairments following damage to the basal forebrain cholinergic system. Brain Research Bulletin, 25, 499-502.

Olton, D. S., Walker, J. A., Gage, F. H. (1978). Hippocampal connections and spatial discrimination. Brain Research, 139, 295-308.

Poucet, B. (1989). Object exploration, habituation, and response to a spatial change in rats following septal or medial frontal cortical damage. Behavioral Neuroscience, 103, 1009-1016.

RifkKinen, P., JR., Sirviö, J., RiekKanen, P. (1990). Similar memory impairments found in medial septal-vertical diagonal band of Broca and nucleus basalis lesioned rats: Are memory defects induced by nucleus basalis lesions related to the degree of non-specific subcortical cell loss? Behavioural Brain Research, 37, 83-88.

SAPER, C. B. (1984). Organization of cerebral cortical afferent systems in the rat. I. Magnocellular basal nucleus. Joumal of Comparative Neurology, 222, 313-342.

Segal, M., Greenberger, V., \& PeArl, E. (1989). Septal transplants ameliorate spatial deficits and restore cholinergic functions in rats with a damaged septo-hippocampal connection. Brain Research, 500, 139-148.

Sutherland, R. J., \& Rodriguez, A. J. (1989). The role of the fornix fimbria and some related subcortical structures in place learning and memory. Behavioural Brain Research, 32, 265-277.

Tilson, H. A., Harry, G. J., Mclamb, R. L., Peterson, N. J., Rodgers, B. C., Pediaditakis, P., A Al, S. F. (1988). Role of dentate gyrus cells in retention of a radial arm maze task and sensitivity of rats to cholinergic drugs. Behavioral Neuroscience, 102, 835-842.

Wenk, G. L., Markowsika, A. L., Olton, D. S. (1989). Basal forebrain lesions and memory: Alterations in neurotensin, not acetylcholine, may cause amnesia. Behavioral Neuroscience, 103, 765-769.

(Manuscript received July 22, 1991; revision accepted for publication November 18,1991 .)

\section{Call for Assistance in The Compilation of a History of the Psychonomic Society}

The Governing Board of the Psychonomic Society is pleased to announce that Robert C. Bolles has agreed to serve as the first Historian of the Society.

All members who might have information relevant to this undertaking are invited to send it directly to Dr. Bolles. Founding members and those who attended the early meetings are especially encouraged to record their reminiscences. While Dr. Bolles hopes to collect as much information as possible relevant to the history of the Society, he will concentrate first on the early history.

Dr. Bolles's address is Department of Psychology, University of Washington, Seattle, Washington 98195 (phone: 206-543-2631). 